Cinémas

Revue d'études cinématographiques

Journal of Film Studies

\title{
De l’intermédialité au multimédia : cinéma, médias, avènement du son
}

\section{Rick Altman}

Volume 10, numéro 1, automne 1999

Cinélekta 3

URI : https://id.erudit.org/iderudit/024802ar

DOI : https://doi.org/10.7202/024802ar

Aller au sommaire du numéro

Éditeur(s)

Cinémas

ISSN

1181-6945 (imprimé)

1705-6500 (numérique)

Découvrir la revue

Citer cet article

Altman, R. (1999). De l'intermédialité au multimédia : cinéma, médias, avènement du son. Cinémas, 10(1), 37-53. https://doi.org/10.7202/024802ar
Résumé de l'article

Prenant en considération l'époque de l'avènement du son aux États-Unis, cet article interroge à la fois les différences entre intermédialité et multimédia et les processus qui mènent de l'une à l'autre. Il propose que le passage de l'intermédialité au multimédia comporte systématiquement trois opérations indépendantes mais reliées : la citation, l'exploitation et la séparation. 


\title{
De l'intermédialité au multimédia : cinéma, médias, avènement du son ${ }^{1}$
}

\author{
Rick Altman
}

\begin{abstract}
RÉSUMÉ
Prenant en considération l'époque de l'avènement du son aux Etats-Unis, cet article interroge à la fois les différences entre intermédialité et multimédia et les processus qui mènent de l'une à l'autre. Il propose que le passage de l'intermédialité au multimédia comporte systématiquement trois opérations indépendantes mais reliées: la citation, l'exploitation et la séparation.

\section{ABSTRACT}

Concentrating on Hollywood's conversion to sound, this article analyzes the differences between intermediality and multimedia, as well as the processes that lead from one to the other. The article proposes a new hypothesis regarding the passage from intermediality to multimedia, identifying three independent but related operations : quotation, exploitation, and separation.
\end{abstract}

Cet article pose un problème historique et cherche à en préciser la théorie constitutive. D'une part, je tenterai d'esquisser une réponse à la question suivante: quels furent, à l'époque de l'avènement du parlant, les rapports entre le cinéma et les autres médias? D'autre part, je tâcherai de préciser le vocabulaire que j'emploie - que nous employons tous - pour traiter de ces rapports. Quelle est au juste la différence entre «intermédialité " et "multimédia "?

J'irai droit au cœur de la question théorique. La notion d'intermédialité proprement dite ne désigne pas pour moi un 
simple mélange de médias, tel qu'on peut le voir et l'entendre dans ces spectacles qualifiés à juste titre d'événements "multimédias". Au contraire, en son sens fort, l'intermédialité devrait désigner, à mon avis, une étape historique, un état transitoire au cours duquel une forme en voie de devenir un média à part entière se trouve encore partagée entre plusieurs médias existants, à un point tel que sa propre identité reste en suspens.

Jusque vers 1910, ce qu'on appelle aujourd'hui le cinéma se trouvait dans cette situation foncièrement intermédiatique. Tiraillé entre plusieurs médias cherchant tous à s'approprier sa nouveauté technologique, le cinéma n'allait sortir de l'intermédialité qu'après s'être séparé définitivement de tous ces autres médias. Afin de conquérir son identité, le cinéma dut déserter l'armée du théâtre, le bataillon des vues ou la division photographique.

Pendant la deuxième moitié des années vingt, le cinéma se trouvait à nouveau dans une situation intermédiatique. Tant qu'il était resté muet, il avait côtoyé paisiblement les mots "téléphone", "phonographe", "radio", sûr de lui, de ce qu'il désignait: une projection d'images en mouvement accompagnées d'un piano ou d'un orchestre. Mais en se faisant bruyantes, parlantes, ces images en mouvement changeaient de nature: le spectateur avait affaire à un nouveau phénomène. Dans le passage au sonore, le cinéma allait perdre son nom. S'il avait suffi du seul mot de cinéma pour désigner des images muettes, l'identité sonore du nouveau venu allait entraîner une folle prolifération de ses désignations. Dans sa nouvelle situation intermédiatique, l'étranger serait traité, c'est le cas de le dire, de tous les noms. Ce que certains appelèrent "talkie" ou un "speakie", d'autres le baptisèrent "dramaphone", "cineoral ", "audifilm", "pictovox", "phonoplay" (nom gagnant du concours sponsorisé par la revue Photoplay), ou même "audien" (le choix de la revue Exhibitors Herald). Docile, peu susceptible, le nouveau venu répondait présent à tous les appels. Il n'habitait nulle part et se logeait à n'importe quelle enseigne. Sans domicile fixe, sans identité fixe. C'est là l'intermédialité telle que je l'entends.

Pour nous, Le Chanteur de jazz est un film, mais comme en témoigne notre première illustration, Le Chanteur de jazz est 


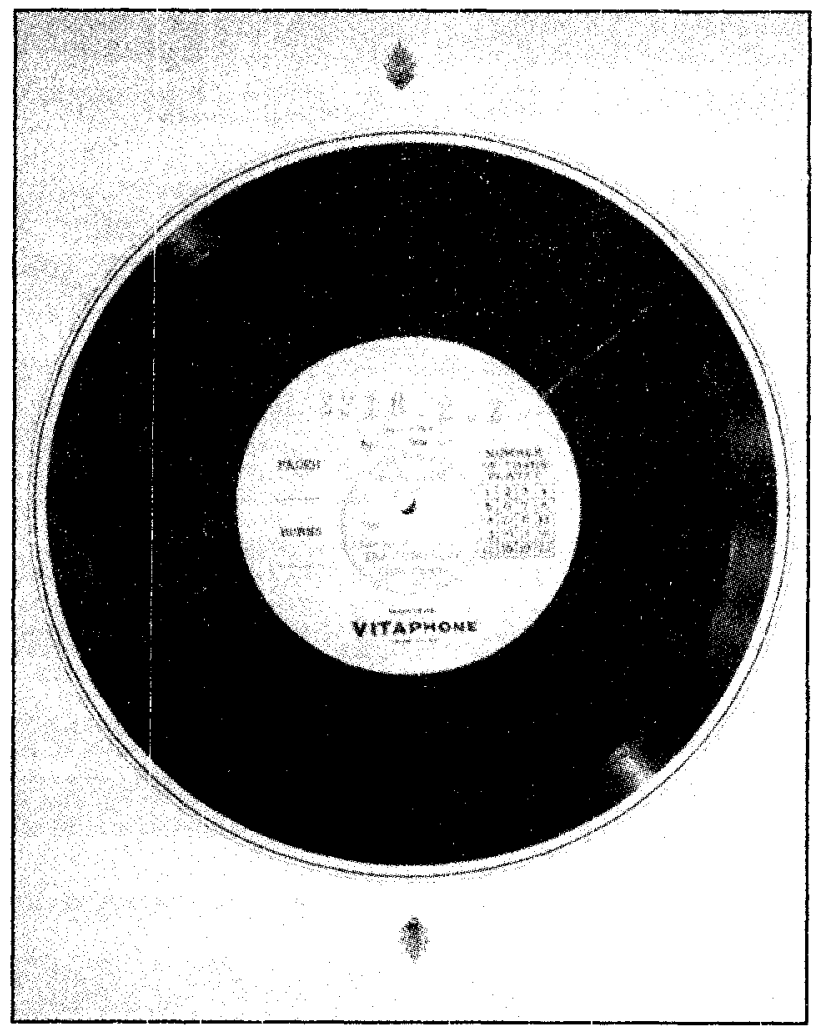

\section{Illustration 1. Disque Vitaphone de la piste sonore du film The Jazz Singer (Warner, 1927)}

d'abord un disque et relève donc de la phonographie. C'est justement ce que notait la critique de l'époque. Pour l'un des commentateurs, "Le film est un simple enregistrement de Jolson sur disque Vitaphone élargi» (Spargo). "Vous voulez ma définition d'un film parlant?, demande un deuxième, c'est un disque phonographique fortement amplifié " (Ennis, p. 43).

Ce ne fut là qu'une des nombreuses définitions qui circulèrent alors. Comme le suggère ce schéma d'une installation Vitaphone de 1927 (illustration 2), la nouvelle technologie n'était pour certains qu'un moyen pour reproduire automatiquement le dispositif du cinéma muet avec son accompagnement musical, et cela sans débourser aucun cachet: un haut-parleur pouvait remplacer avantageusement les musiciens dans la fosse d'orchestre.

De l'intermédialité au mulrimédia : cinéma, médias, avènement du son 


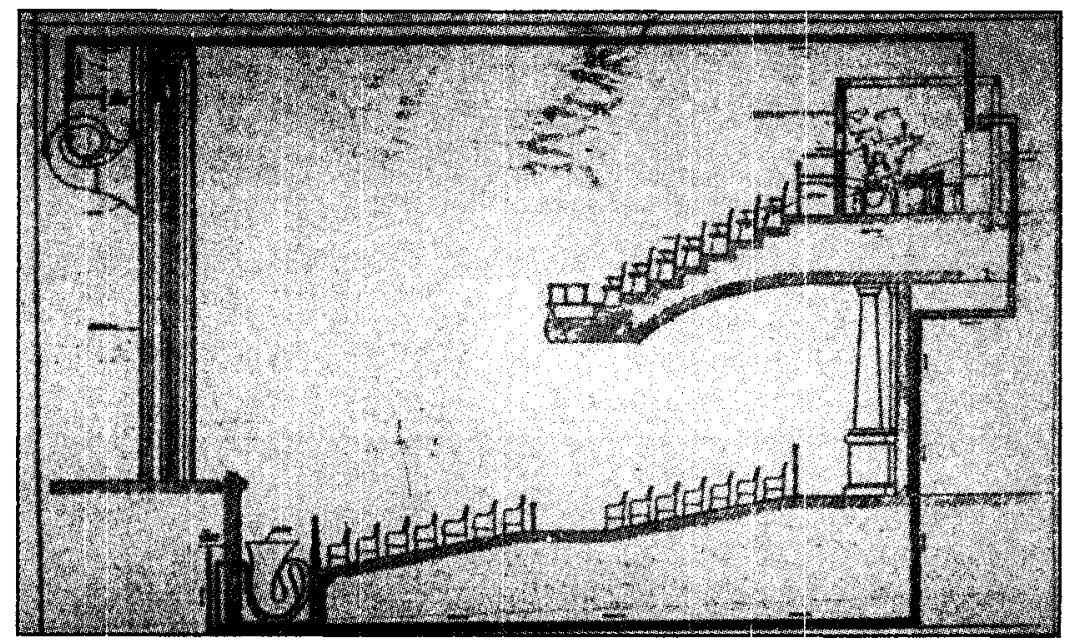

\section{Illustration 2. Vue schématique d'une installation d'un système sonore Vitaphone de 1927, provenant de H. M. Wilcox, " Data for projectionists on operation of Vitaphone ",, Exhibitors Herald (9 mai 1927), p. 11}

Mais voilà que je parle de "haut-parleur". Alors que pour les gens de chez Western Electric, il s'agissaient plutôt d'un "téléphone parlant haut" ("loudspeaking telephone»). On ne saurait leur donner tort, car pendant les années vingt, le seul hautparleur connu par le commun des mortels était justement le récepteur du téléphone. Comme le révèlent les illustrations 3 et 4, les inventeurs du Vitaphone étaient redevables à la technologie et au financement téléphoniques: ils avaient donc tout intérêt à ce que le nom donné à leur création rappelle celui de leur "patriarche": Bell Telephone.

Mais le groupe téléphonique AT\&T-Bell-Western Electric ne fut pas le seul prétendant au titre de parrain. Il y avait également la Radio Corporation of America - la puissante RCA, avec ses stations de radio, son studio RKO et ses Radio Pictures. Albert Warner lui-même n'avait-il pas calqué le premier programme Vitaphone d'août 1926 sur une émission radiophonique à grand succès diffusée chaque dimanche soir par la maison de disques Victor $^{2}$ ? L'imagination populaire ne supposait-elle pas que les 


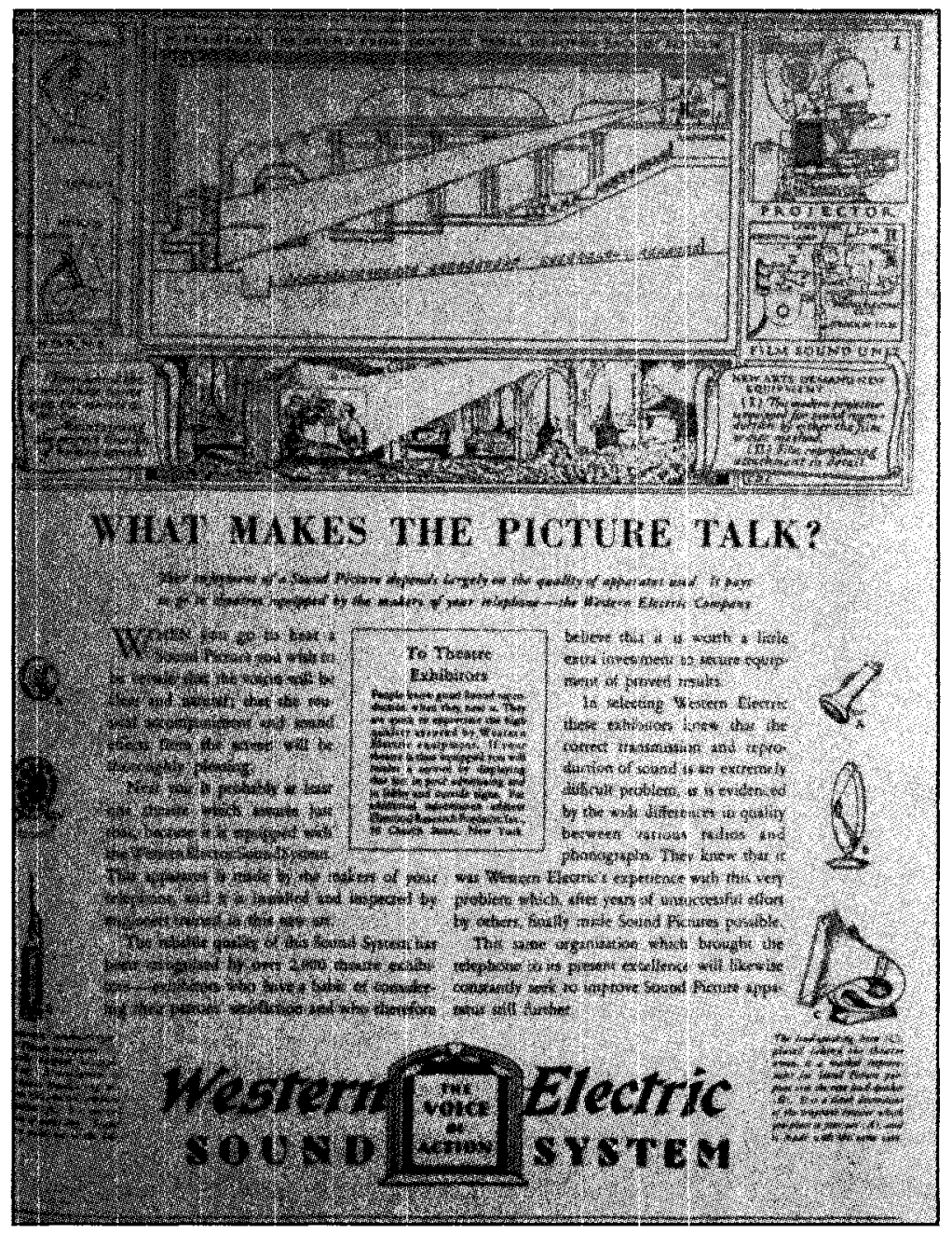

\section{Illustration 3. Publicité de la Western Electric, provenant du Saturday Evening Post (13 juillet 1929), p. 111}

paroles d'un film sont produites par ce qu'on a pu appeler « [...] un émetteur radiophonique miniature perché dans la salle de projection ${ }^{3}$ "? On ne s'étonnera donc pas en apprenant que la RCA, dans une campagne publicitaire, s'est ingéniée à représenter ses films comme des émissions de radio (illustrations 5, 6 et 7), tout comme AT\&T et ses filiales avaient traité leur

De l'intermédialité au multimédia : cinéma, médias, avènement du son 


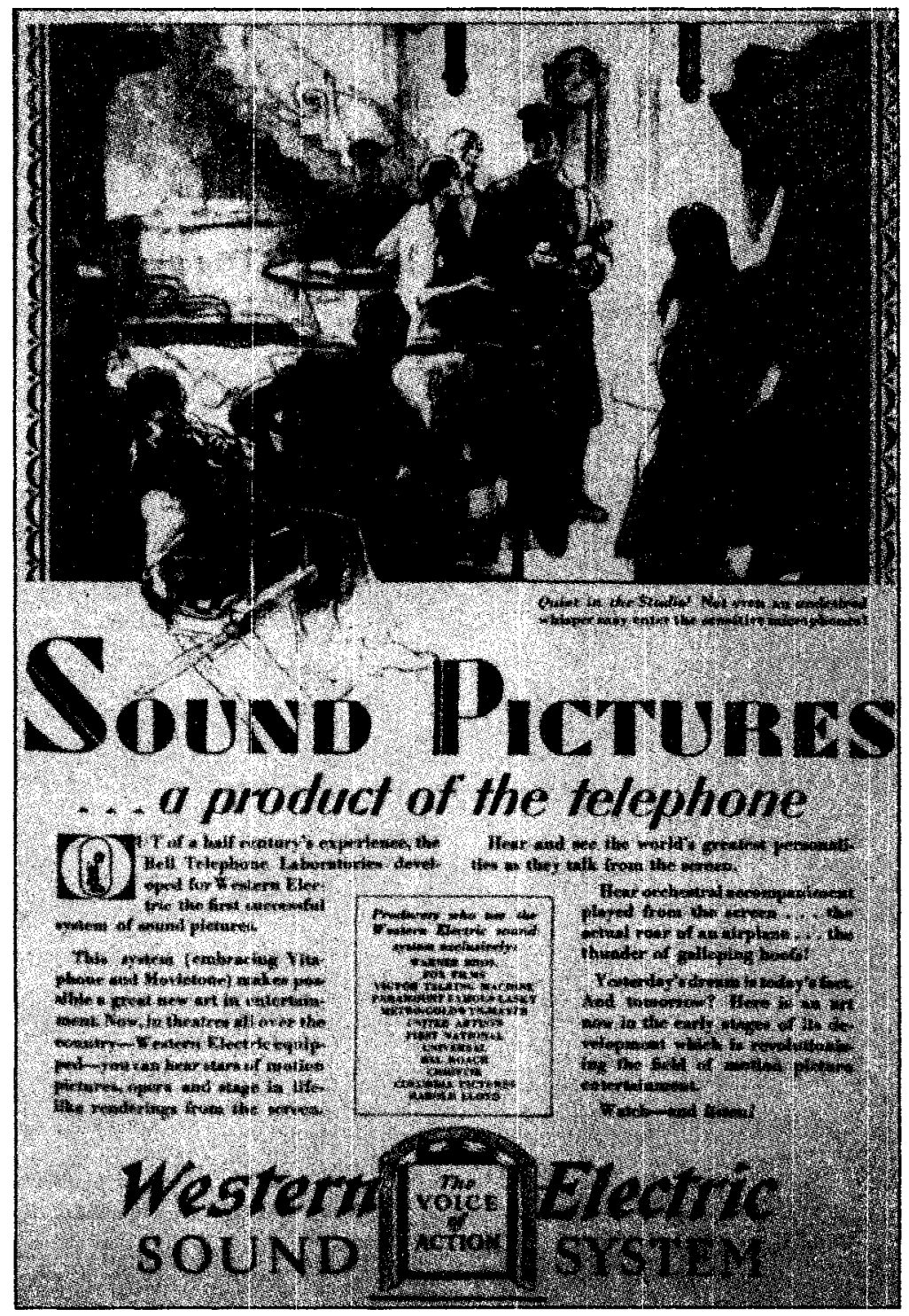

\section{Illustration 4. Publicité de la Western Electric, provenant de Life ( \pm 1929)}

nouvelle technologie audiovisuelle comme une simple extension de la téléphonie.

Cette situation foncièrement intermédiatique a empêché pendant plusieurs années la constitution du média simple et 


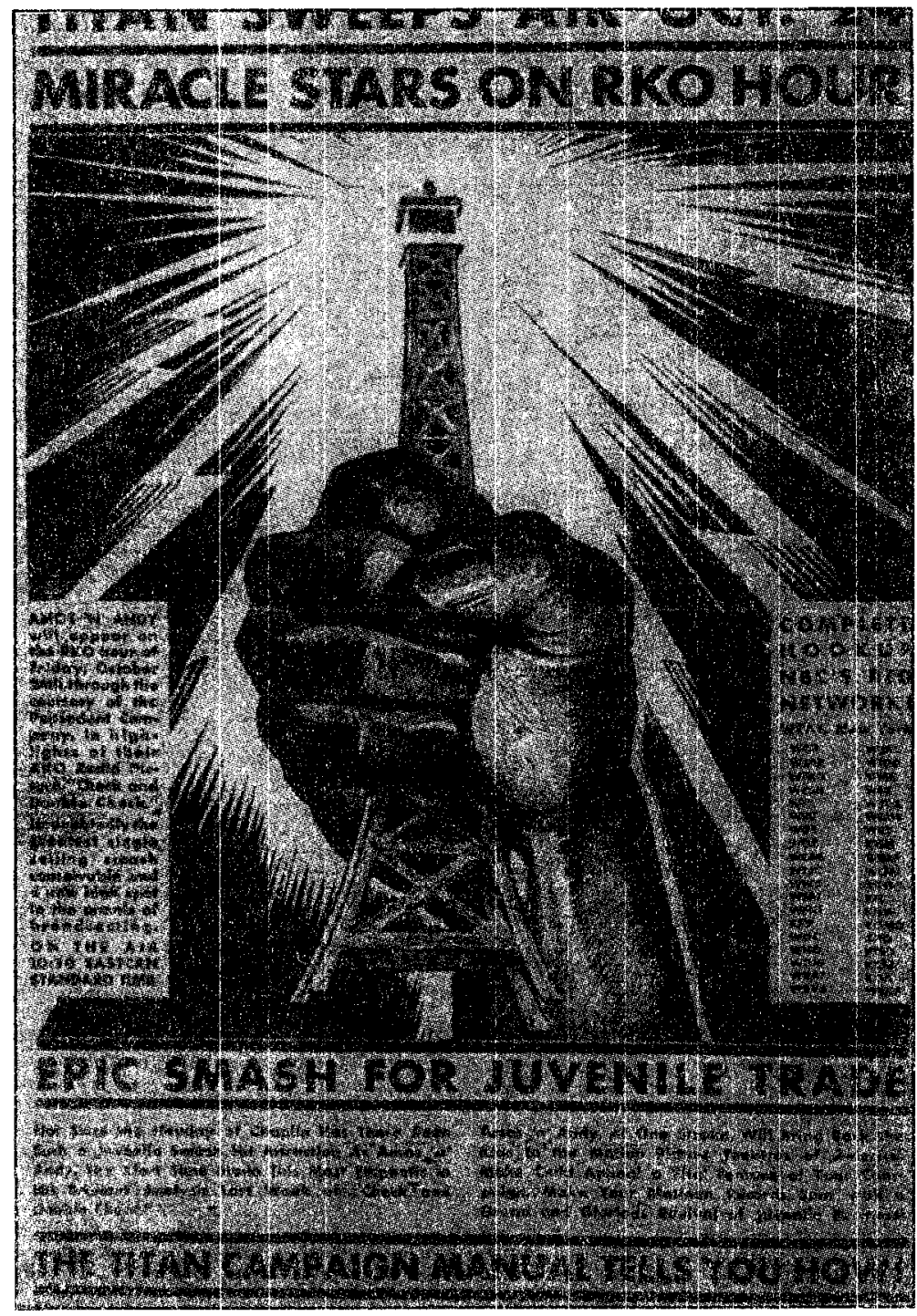

\section{Illustration 5. Publicité de la RKO, provenant de Variety (23 octobre 1929), p. 34-35}

relativement stable que, depuis les années trente, nous appelons à nouveau "le cinéma" tout court. Une fois reconstitué, redéfini, rebaptisé, c'est-à-dire sorti de l'intermédialité, le cinéma gagnera l'indépendance qui lui permettra par la suite d'être souvent mêlé à des représentations qu'on a raison de qualifier de 


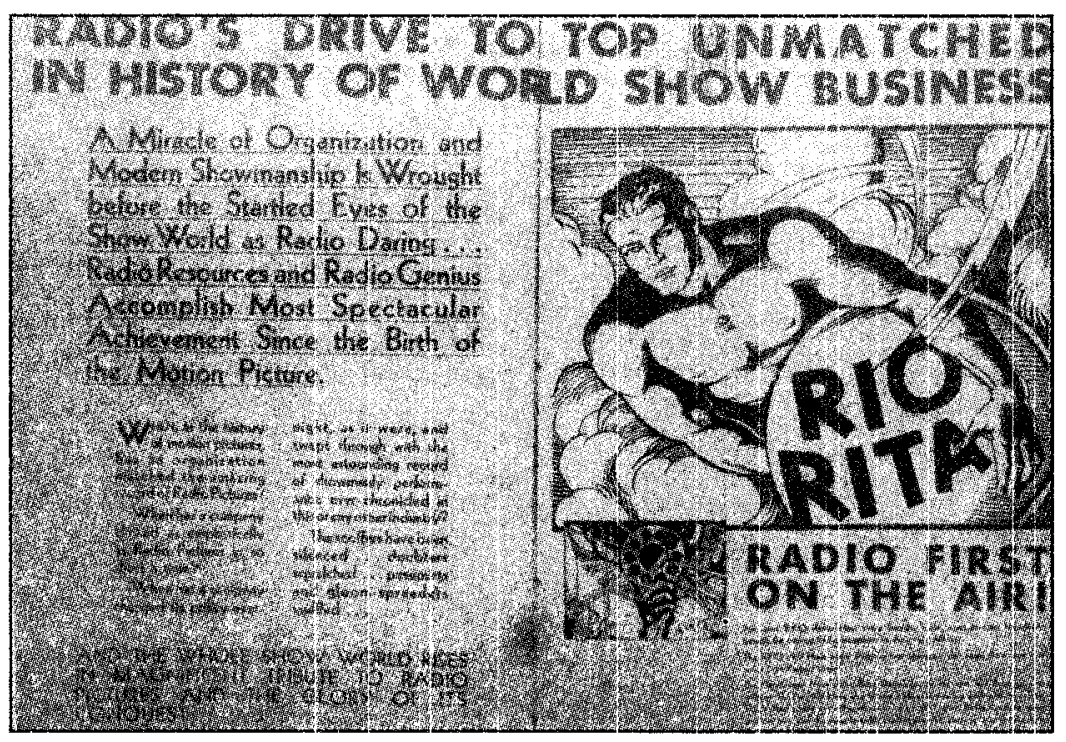

\section{Illustration 6. Publicité de la RKO, provenant de Variety (18 juin 1930), p. 34-35}

"multimédia", étant donné le caractère désormais stable des médias en question. Si l'intermédialité suppose une forme prise entre plusieurs médias, une forme dont l'identité reste en suspens, le multimédia dépend de l'existence de médias assez confiants en leur identité respective pour oser les combinaisons les plus outrées.

Mais comment l'intermédialité de 1927 est-elle devenue le multimédia de 1935? Quels sont les moyens adoptés par le cinéma pour sortir de son anonymat intermédiatique? Bref, comment passe-t-on de l'intermédialité au multimédia? Quelle est la logique de ce passage, et quels en sont les procédés? Ce sont là des questions d'une importance capitale et qui n'ont malheureusement jamais été clairement posées. Cependant, il s'agit de questions qui dépassent largement le cadre d'un seul article. On ne résoudra pas ces problèmes en quelques lignes. Mais faisons tout de même un premier pas: offrons une hypothèse qui aura le mérite tout au moins de lancer la discussion.

Je propose de reformuler l'histoire traditionnelle de l'avènement du son en considérant essentiellement le devenir de ce média qu'on appelle aujourd'hui "cinéma", sans jamais se 


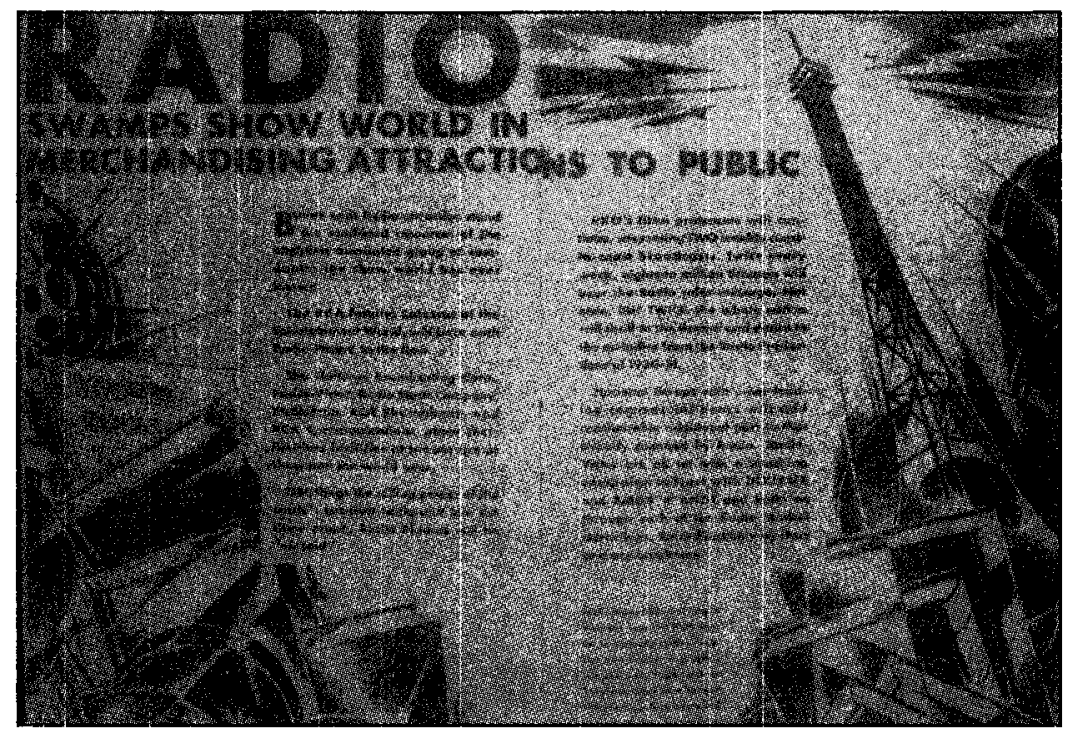

\section{Illustration 7. Publicité de la RKO, provenant de Variety (15 octobre 1930), p. 18}

douter que le mot et son référent ne furent pas toujours aussi solidaires qu'il nous le semble maintenant. Certains ont vu l'avènement du son comme l'histoire tripartite de l'invention, de l'innovation et de la diffusion ${ }^{4}$. Selon mon hypothèse, c'est à un autre trio qu'il faut recourir. Le cinéma se libère d'un stade intermédiatique en mettant en pratique trois procédés: la citation, l'exploitation et la séparation. Apparaissant parfois comme des étapes successives plutôt qu’à titre de procédés indépendants - je renforcerai malheureusement cette tendance par mon mode de présentation -, la citation, l'exploitation et la séparation sont de fait liées logiquement plutôt que chronologiquement. Elles constituent autant de façons de garantir l'existence du cinéma comme espèce médiatique indépendante. Dans la logique, c'est cet ordre-là qui leur est naturel, mais dans la nature l'ordre chronologique n'est guère toujours respecté.

Anonyme et apatride, la nouvelle technologie se met en quête d'un nom, d'un pays. Quel meilleur moyen pour s'assurer une identité que d'emprunter celle d'autrui? C'est exactement ce que fait la technologie bâtarde en citant un média solidement établi. Mais elle ne saura résister à la tentation de multiplier ses 
chances en citant tour à tour plusieurs médias. Ce procédé au moyen duquel une nouvelle forme cherche à tout prix à se faire accepter, mais en citant successivement plusieurs médias, constitue un indice: il révèle une situation intermédiatique intense. Plongée dans cette situation, la nouvelle technologie n'arrive qu'à renforcer son anonymat.

Voici quelques exemples de cette stratégie de citation intermédiatique. Il s'agit de films ou d'extraits de films peu connus, produit entre 1923 et 1929 , mais il va de soi que ce que j'appelle la citation représente moins une étape première au plan chronologique qu'une opération préliminaire à l'exploitation et à la séparation au plan logique.

Cohen on the Telephone. Ce sketch Phonofilm de Lee De Forest (1923) cite tout bêtement un numéro de vaudeville existant, ce dernier citant lui-même une conversation téléphonique. Ce film identifie le cinéma parlant au téléphone, car l'impossibilité pour Cohen de voir le gérant de son immeuble tandis qu'il converse avec lui est attribuée au fait que le téléphone ne permet pas une telle prouesse.

The Voice on the Screen. Ce film a été réalisé en 1926 par les laboratoires Bell, inventeurs du procédé Vitaphone, pour un colloque de spécialistes. Tout comme les Phonofilms de De Forest cherchaient une identité à travers leur capacité à remplacer un numéro de variétés, ce film industriel remplace intégralement le discours que le directeur Craft devait prononcer au colloque. Selon Craft, la nouvelle forme n'est qu'une extension de la téléphonie et de la sonorisation des discours publics, deux techniques popularisées par le groupe AT\&T-Bell-Western Electric que Craft représente.

Introduction to the Vitaphone. Cette première partie du programme de courts métrages Vitaphone, qui a précédé la projection de Don Juan en août 1926, met en scène Will Hays, Chairman du Motion Picture Producers and Distributors Association. Quel paradoxe que cette conférence! Alors qu'en paroles, Hays présente et explique la nouvelle technologie, ses gestes font de l'exposé un discours public à l'ancienne. Mais comment démontrer l'utilité du nouveau média en 1926, sinon en faisant valoir qu'il est capable de tout ce que font les autres 
médias. La stratégie n'a pour seul défaut que de trop bien réussir, le nouveau rnédia se voyant assimilé à l'ancien.

Overture to Don Juan. Après les sujets courts de ce même programme de 1926, on aborde le long métrage de la soirée: dix minutes (une bobine entière) de musique sur fond d'écran noir. Cela est-il vraiment du cinéma? Peut-être pas pour nous, mais à l'époque, la survie même de la nouvelle technologie dépendait de sa capacité à remplacer les technologies existantes, d'où cette citation directe de l'orchestre de cinéma muet.

Radio Rhythrn. Déjà en 1929, le cinéma se mettait à citer son nouveau concurrent, la radio. Or, Radio Rhythm reproduit exactement l'émission de Rudy Vallee. Plutôt que de faire voir les coulisses ou de commencer medias res, ce petit film démarre par le fameux "Hi-ho everybody", signature orale de Rudy Vallee et point de départ habituel de son émission.

Le but de ces citations très variées - et j'en passe - est de toute évidence de stabiliser l'identité du cinéma. Chaque citation se veut une preuve de ce que le cinéma est capable de tout ce que peut un autre média $:$ cinéma $=$ média $x$. Mais paradoxalement, se voulant égal aux autres, le cinéma en vient à prouver qu'il lui manque une identité propre. Il finira donc par comprendre qu'il lui faut à tout prix exploiter les autres médias plutôt que de les citer naïvement.

Cette deuxième stratégie ne cherche donc plus à identifier le cinéma aux autres médias, mais à se servir de médias concurrents comme de partenaires qui lui feraient la courte échelle. Le cinéma invoquera donc de nouveau les autres médias, mais cette fois pour démontrer, plus ou moins ouvertement, sa supériorité. Voici quelques exemples qui mettent en évidence cette exploitation.

Ramblin' Round Radio Row. Ce film Vitaphone de 1932 représente le degré zéro de cette deuxième stratégie. Si la radio qui paraît à l'écran ne sait offrir que de la musique, le cinéma, lui, a le pouvoir de fournir l'image qui lui est associée, comme nous le prouve la suite de ce petit film.

Red Nichols \&r His Five Pennies. Ce film remet à l'honneur une technologie qu'on aurait crue oubliée bien avant les années trente. Le programme tout entier passe d'un kinétoscope à 
l'autre, le cinéma leur conférant le pouvoir de nous transporter dans un autre lieu et d'agrandir l'image.

Rhapsody in Black and Blue. Dans ce film de 1932, le cinéma fait valoir de nouveau son pouvoir de transporter le spectateur dans un royaume que ne sauraient atteindre les auditeurs de tout autre média. Symboliquement, le disque phonographique qu'écoute et accompagne le pauvre mari finira par être cassé, alors que grâce au cinéma, on accédera en un lieu céleste où la trompette sublime de Louis Armstrong remplace les casseroles du mari.

Three's a Crowd. Un tel procédé d'exploitation est loin d'être limité au cinéma photographique, il est aussi utilisé par le dessin animé, comme le prouve ce petit film d'animation de 1932. Isolées, la littérature reste muette et la radio, aveugle: mais le dessin animé, rythmé et parlant permer de combiner paroles, musique et images - preuve indubitable de la supériorité du cinéma parlant.

Cette exploitation des autres médias vise systématiquement à montrer que le cinéma est capable de prouesses qui dépassent les capacités des autres médias : cinéma $>$ média $x$. L'image du kinétoscope est toute petite, la radio et la phonographie n'ont pas d'images, les livres n'ont pas de son, etc. Mais cherchant à tout prix à proclamer sa supériorité sur les autres médias, le cinéma met un certain temps à définir et à explorer les possiblités qui lui appartiennent en propre. Bien sûr, il offre à la fois son et image, mais les exploite-t-il de façon à se démarquer?

À partir de 1929, on trouve de plus en plus de scénarios et de situations qui semblent créés pour prouver que le cinéma est fondamentalement différent des autres médias. En voici un échantillon.

Say It with Songs. Plus que Le Chanteur de jazz de 1927, c'est Le Fou chantant de 1928 qui a fait la réputation d'Al Jolson. Son troisième film, Say It With Songs (1929), était donc particulièrement attendu. Chanteur à la radio, $\mathrm{Al}$ est marié à une femme qui l'adore et qui repousse donc les avances de son producteur. Malheureusement, l'explication entre $\mathrm{Al}$ et le prétendu séducteur tourne mal et le producteur meurt d'une chute. Al adore son mioche, l'omniprésent Davey Lee, qui à plusieurs reprises contribue sans le vouloir à inculper son père. À peine sorti de prison, $\mathrm{Al}$ 
est responsable d'un accident automobile et cause - également sans le vouloir -- des blessures à son fils. Le petit mignon finit par se rétablir, mais ne parvient pas à retrouver la voix. Là où la médecine échoue, là où la radiophonie et la phonographie font faillite, le $7^{\circ}$ art fera des miracles. Grâce à lui, l'enfant recouvre l'usage de la parole. Et grâce à la technique de la surimpression, le cinéma transporte les spectateurs au septième ciel.

Dans un sens, Say It With Songs est une simple extension du backstage musical, faisant voir non seulement la scène et ses coulisses, mais aussi l'un après l'autre le lieu de l'émission et le lieu de l'audition, le temps de l'émission et ceux de la préparation et de la réaction. C'est là une technique qui sera de plus en plus exploitée par un cinéma de plus en plus conscient de sa souveraineté. Se considérant limité par le téléphone, Cohen savait qu'il ne pouvait "voir" son gérant: nous autres, spectateurs, n'y avions donc pas droit non plus. Dans Say It With Songs, nous voyons les deux interlocuteurs, chacun dans son espace: nous voyons non seulement le chanteur radiophonique au studio, mais aussi ses auditeurs chez eux: et le son d'un disque, enregistré bien avant, invoque en surimpression l'image du chanteur. C'est en insistant sur un tel redoublement du temps et de l'espace - technique spécifiquement cinématographique - que le cinéma réussira à se séparer définitivement des autres médias avec lesquels il avait été auparavant confondu.

Voices of Hollywood. Alors que certains films ne font qu'exploiter les autres médias afin de proclamer la supériorité du cinéma, un nombre croissant de films, petits et grands, seront conçus comme des divertissements multimédias, insérant la radio, le téléphone, et même les marionnettes dans un cadre proprement cinématographique. On trouve des exemples particulièrement frappants de cette technique dans la série Voices of Hollywood, produite en 1929. Alors que Cohen on the Telephone renforçait l'identité du cinéma et de la téléphonie en identifiant le début et la fin du film au début et à la fin de l'appel téléphonique, Voices of Hollywood commence à la fois avant le coup de fil et avant l'émission radiophonique représentée dans le film. Grâce au cinéma, nous voyons non seulement radio, téléphone et marionnettes, mais aussi le hors-scène de chaque 
média ainsi que le hors-micro et le hors-temps de chaque émission. Plus encore, le cinéma sert désormais de coordonnateur essentiel entre les médias. Le comique de cette saynète provient justement de la possibilité de mélanger plusieurs médias, mais aussi et surtout, des conflits suscités par ce mélange.

Le rôle de coordination assuré par le cinéma est d'une importance capitale dans ces films multimédias qui pullulent après la découverte du play-back en 1929 et qui exploitent l'écart entre la voix et son enregistrement, entre le corps audible et le corps visible. Les deux derniers exemples relèvent justement de cette catégorie. Produits par Mack Sennett et mettant en vedette Bing Crosby, ce sont des courts métrages qui méritent d'être mieux connus.

I Surrender Dear. Rivalisant avec Rudy Vallee pour le titre de chanteur préféré de la gent féminine, Bing Crosby s'est mis en 1931 à faire une série de films qui malgré leur courte durée ne manquent pas d'innovations multimédias. Tombé subitement amoureux d'une "belle jeunesse", Crosby doit prouver dans ce film que la voix qu'on entend à la radio est bien la sienne. Comme maint autre film de l'époque, la trajectoire de $I$ Surrender Dear part d'une voix médiatisée et mal identifiée à son auteur pour finir sur une série de scènes où - la médiatisation de la phonographie ou de la radio disparue - la voix et son auteur sont enfin réunis, permettant ainsi la réunion du chanteur et de sa bien-aimée.

Sing Bing Sing. Dans ce court métrage, également de 1931, Sennett et Crosby redéfinissent une fois pour toutes le statut du cinéma. On commence par un message radiophonique personnel entre les jeunes amoureux, mais ce message est intercepté par "papa». Changeant de tactique, on passe au téléphone: manque de pot, "papa" est toujours à l'écoute. Heureusement que la phonographie est là pour leurrer "papa» et ses gorilles: Bing et sa bien-aimée n'échapperont à l'emprise paternelle qu'en substituant la voix enregistrée de Bing à sa vive voix. Ce sont là, effectivement, autant de médias (radio, téléphone, phonographe) sur lesquels on ne saurait compter. Seul le cinéma mérite pleinement notre confiance, lui qui est identifié, dans la dernière scène, d'une part à l'aéronautique et donc à la moder- 
nité, d'autre part au discours direct non médiatisé qu'est celui de l'amour. Pour la énième fois, on se trouve en fin de course dans les nuages, comme si seul le $7^{\circ}$ art était capable de nous emmener au septième ciel: par sa capacité de dépasser les limites de tout autre média; par son usage des surimpressions, des images composites et de l'animation; par son prétendu contact direct, entre chanteur et auditrice, entre film et spectateur.

Ce perit film offre un bel exemple de notre troisième et dernière étape, celle de la séparation des médias, qui prouve par $a$ plus $b$ que le cinéma n'est pas égal à média $x$. Il faut admirer le travail effectué par le cinéma pour se forger une identité aussi forte que celle des médias de la vieille noblesse. Car il s'agit bel et bien d'un travail. Confondu en 1927 avec le téléphone, la radio et la phonographie - autant de médias bénéficiant du soutien financier d'imposantes entreprises -, le cinéma assumera quelques années plus tard le rôle de coordonnateur des autres médias. Naguère identifié à ces autres médias, se cachant pour ainsi dire sous leurs jupons, le cinéma sera dorénavant séparé des autres médias, identifié à ses propres capacités et à ses propres techniques, plutôt qu’à celles qu'il partage avec les autres.

Rappelons que tout au long des années trente, le cinéma continuera à partager une importante base technologique et économique non seulement avec le théâtre, le téléphone, la radio, la phonographie et la sonorisation des discours publics, mais aussi avec ce nouveau média qu'aujourd'hui on appelle la télévision.

D'ailleurs, je note en passant, que pour aborder l'histoire de la télévision des premiers temps, il serait nécessaire de revenir non seulement à l'intermédialité - car la télévision fut longtemps confondue avec d'autres médias, si bien qu'on l'a longtemps et tout simplement appelée "radio" -, mais aussi à la triade que je viens de présenter: citation, exploitation et séparation. Car le système proposé ici ne se limite pas au seul cinéma : au contraire, il s'applique au double mouvement d'inscription et d'effacement de l'intermédialité propre à l'introduction de toute nouvelle technologie, à l'apparition de toute nouvelle catégorie sociale.

Lordinateur, par exemple, n'a d'abord été qu'une machine à écrire améliorée ou encore, une calculatrice plus performante 
que les autres. Les premiers logiciels se contentaient donc d'imiter, de citer les formes déjà employées par les écrivains, les comptables ou les scientifiques. Ce n'est qu'à travers une comparaison entre ses propres capacités et celles des autres médias que l'ordinateur se met à exploiter sa propre identité. Aujourd'hui, grâce à ses capacités graphiques et à son potentiel d'interconnexion, l'ordinateur se sépare nettement de ses concurrents. Mais attention, l'Internet, est-ce la même chose que l'ordinateur? Non, si l'on juge de la capacité de l'Internet - du moins dans la phase adolescente où il se trouve actuellement à citer à outrance les autres médias, au point de faire se confondre l'ordinateur et la télévision sur des sites de "Web TV".

Il ne suffit point - ce dernier exemple le confirme - de tracer le passage de l'intermédialité au multimédia, car une nouvelle intermédialité nous attend toujours au détour. Affranchi aux alentours de 1930 de sa large famille intermédiatique - papa Ampli, maman Micro, sœur Sono, frangin Phono, tonton Téléphone, tata Radio - , le cinéma se trouva bientôt plongé par la télévision dans une nouvelle crise intermédiatique. Au tour de la télévision aujourd'hui de se trouver compromise par l'intermédialité informatique.

Mais c'est là une tout autre histoire, qui devra attendre son historien. Pour le moment, ne faut-il pas d'abord passer par une discussion portant à la fois sur la théorie de l'intermédialité et sur une tranche d'histoire des médias sonores, la riche histoire de l'avènement du son au cinéma?

University of Iowa

NOTES

1 Ce texte reprend les principaux éléments d'une conférence prononcée en octobre 1998 à la Cinémathèque québécoise (Montréal), dans le cadre de l'événement Résonances et contrepoints, organisé conjointement par la Phonothèque québécoise et l'Association québécoise des études cinématographiques, en collaboration avec le Centre de recherche sur l'intermédialité (Université de Montréal).

2 Voir à ce sujet, "Perfect Automatic Music for Movies" (Times, 26 avril 1926, p. 7).

3 Ruth Russell, "Voice Is Given to Shadows of Silver Screen" (Tribune, 16 septembre 1926). 
4 Je pense particulièrement aux travaux de J. Douglas Gomery. Pour une explication de la méthode de Gomery, voir Robert C. Allen et Douglas Gomery, Film History: Theory and Prattice (New York: Alfred A. Knopf, 1985), et en particulier le chapitre 5, "Technological Film History" (p. 109-130).

\section{OUVRAGES CITÉS}

Ennis, Bert. "Sophie Goes Talkie: The Last of the Red-Hot Mamas Falls for the Films". Moving Picture Classic, vol. 29, n" 3 (1929).

Spargo, John S. "Jolson's Songs Help The Jazz Singer at New York Premiere". Exhibitors Herald, 15 octobre 1927. 